\title{
Boron isotope calibration of large benthic foraminifera species using LA- MC-ICP-MS
}

\author{
MR. DOUGLAS T COENEN ${ }^{1}$, DAVID EVANS ${ }^{2}$, HAGAR \\ HAUZER $^{3}$, JONATHAN EREZ ${ }^{3}$, LAURA COTTON ${ }^{4}$, \\ WILLEM RENEMA ${ }^{5}$ AND WOLFGANG MÜLLER ${ }^{2}$ \\ ${ }^{1}$ Goethe University \\ ${ }^{2}$ Institute of Geosciences, Goethe University \\ ${ }^{3}$ The Fredy \& Nadine Herrmann Institute of Earth Sciences, the \\ Hebrew University of Jerusalem \\ ${ }^{4}$ School of the Environment Geography and Geosciences, \\ University of Portsmouth \\ ${ }^{5}$ Naturalis Biodiversity Center \\ Presenting Author: coenen@geo.uni-frankfurt.de
}

Constraining Earth's climate sensitivity using past climate reconstructions is one of the key contributions of palaeoclimate data to preparing for ongoing anthropogenic climate change. Precisely determining atmospheric $\mathrm{CO}_{2}$ concentrations during past key warm intervals such as the early-middle Paleogene is an ideal target in this respect. Of the available palaeo- $\mathrm{CO}_{2}$ proxies, the boron isotopic composition of marine carbonates is a key method of producing quantitative $\mathrm{pH} / \mathrm{CO}_{2}$ reconstructions, yet data are lacking, or available at low resolution for parts of the Eocene.

The boron isotope $\mathrm{pH}$ proxy generally relies on empirical calibrations to relate the measured boron isotopic composition of the foraminifera to the boron isotopic composition of the aqueous borate ion in the paleo-ocean. Empirical calibration of planktonic foraminifera has shown that species or group-specific vital effects are present, with the implication that the application of the proxy to lineages with only indirect descendants is associated with some uncertainty.

To address this, we present an empirical calibration for an under-utilised group that was very abundant during the Paleogene, the shallow-dwelling large benthic nummulitid foraminifera (LBF, specifically Operculina ammonoides). An advantage of this group is that Operculina has a lineage extending back to the early Paleogene, and is closely related to the widespread Eocene Nummulites. Our calibration is based on measurements of samples grown under a range of carbonate chemistries in laboratory culture, spanning a $\mathrm{pH}$ range of $\sim 7.6$ to 8.4. We use LA-MC-ICPMS as analytical technique for which LBFs are particularly well-suited and demonstrate that samples can be characterized to a precision of up to $0.3 \%$ (typically $<0.5 \%$, 2SE, $\mathrm{n}=20-50$ ) when using $10^{13} \Omega$ resistors and a 40 $\mu \mathrm{m}$ laser spot. This combination of using a so far underutilized biomineral archive, a robust calibration and spatially-resolved $\delta^{11} \mathrm{~B}$-measurements paves the way towards an improved palaeo$\mathrm{pH} / \mathrm{pCO}_{2}$ reconstruction for the Paleogene, with implications for understanding Earth's climate sensitivity during past warm intervals. 